\title{
Spin Relaxation in InSb and InAs by 1- and 2-Colour Spectroscopy with Free Electron and Solid State Lasers
}

\author{
C. R. Pidgeon ${ }^{1}$, P. Murzyn' ${ }^{1}$, P. J. Phillips ${ }^{1}$, B. N. Murdin², K. Litvinenko' ${ }^{2}$, M. Merrick ${ }^{2}$, L. F. Cohen ${ }^{3}$, \\ T. Zhang ${ }^{3}$, S. K. Clowes ${ }^{4}$, P. Buckle ${ }^{4}$ and T. Ashley ${ }^{4}$. \\ 'Department of Physics, Heriot-Watt University, Edinburgh EH14 4AS, UK \\ ${ }^{2}$ Advanced Technology Institute, University of Surrey, Guildford GU2 7XH, UK \\ ${ }^{3}$ Blackett Laboratory, Imperial College of London, London SW7 2BW, UK \\ ${ }^{4}$ QinetiQ, St Andrews Road, Malvem, Worcs WR14 3PS, UK
}

\begin{abstract}
We report time-resolved measurement of spin lifetimes in both bulk and quantum well narrow gap semiconductors (NGSs) at $300 \mathrm{~K}$. We have used both free electron and solid state laser sources from 3 to $7 \mu \mathrm{m}$ and quarter wave plates to produce circularly polarised pump and probe beams. We previously demonstrated that spin flip (Elliott-Yafet, EY) scattering dominates in $\mathrm{Hg}_{0.78} \mathrm{Cd}_{0.22} \mathrm{Te}$ due to strong lattice scattering. In contrast, spin unphasing (D'Yakonov-Perel, DP) scattering controls the loss of polarisation in intrinsic InSb and InAs due to the higher mobility, giving spin lifetimes, $\tau_{\mathrm{s}}$, of $\sim 20 \mathrm{ps}$ at $300 \mathrm{~K}$. We report first measurements of $\tau_{\mathrm{s}}$ of $\sim 17 \mathrm{ps}$ in InSb/InAlSb quantum wells.
\end{abstract}

\section{Introduction}

Utilisation of the electron spin has become a focus of interest in semiconductor electronics, or spintronics, in recent years. On the one hand it is important to realise a sufficiently long spin lifetime to process information stored in the form of the polarisation of spin ensembles [1-3]. But in addition, to control the lifetime it is necessary to understand the spin relaxation mechanisms in both bulk and low dimensional semiconductor structures which are to be designed so that spins can be appropriately confined and/or transferred. Narrow gap semiconductors (NGSs) are interesting for spintronic applications in general because of their high electron $\mathrm{g}$-factor, Rashba splitting (enabling lifetime control by extenernal electric fields) and mobility.

In bulk NGSs two main spin relaxation processes have been found to be important: D'yakonov-Perel [DP] [4] and ElliottYafet [EY] [5].

For DP the macroscopic spin relaxation is due to an unphasing of the spins. The eigenstates of the spin-orbit interaction are not pure spin up or down, but a linear combination of the two, and are spin-split at non-zero $\mathbf{k}$ by inversion asymmetry (which gives terms proportional to $\mathrm{k}^{3}$ in the conduction band). Injection of spin polarised electrons from a magnetic material (or creation by circularly polarised light) gives a linear combination of the eigen-states whose phase evolves with time at a rate given by the spin splitting. This is equivalent to the spins precessing about a pseudo-field, $\mathbf{B}_{\mathrm{eff}}$, whose strength and direction are dependent on the magnitude and direction of $\mathbf{k}$. In an ensemble of electrons of different $\mathbf{k}$, the macroscopic polarisation unphases (the DP mechanism). In the presence of momentum scattering DP is subject to motional narrowing, i.e. electron collisions cause $\mathbf{k}$, and hence $\mathbf{B}_{\mathrm{efr}}$, to perform a random walk so slowing the unphasing. Somewhat counter-intuitively therefore, $\tau_{s}(\mathrm{DP})$ is proportional to the inverse of the orbital momentum scattering time, $\tau_{\mathrm{p}}$. [NB $\tau_{\mathrm{p}}$ is not necessarily equal to the mobility time as the latter is not sensitive to electronelectron scattering ]

In the presence of spin-orbit coupling EY showed that, because the spins become mixed in the conduction band through the conduction-valence band interaction, spin flip scattering that transfers angular momentum to the lattice becomes allowed, resulting in a contribution to $\tau_{s}(E Y)$ that is directly proportional to the mobility time, $\tau_{p}$. The DP and EY processes can be distinguished by their differing dependence on mobility.

\section{Experiment and results}

We have made time resolved measurements of spin lifetimes in bulk and quantum well NGSs, using free electron and solid state laser sources from $3-7 \mu \mathrm{m}$ and quarter wave plates to produce circularly polarised pump and probe beams. In these experiments a circularly polarised interband pump pulse creates spin-polarised electrons. A circularly polarised probe pulse then measures induced circular dichroism in the transmission due to the band filling. The probe can be polarised with the same circular polarisation (SCP) or opposite circular polarisation (OCP) to the pump, and the ratio of the difference divided by the sum of the probe transmission determines the spin polarisation, $\mathrm{P}=(\mathrm{SCP}-\mathrm{OCP}) / \mathrm{SCP}+$ $O C P$ ), at delay times after the pump pulse. This measurement is rather difficult when the time constants involved are very short and comparable to the optical thickness of the sample as muitiple reflections can cause discontinuities in the data. It is also more difficult when the spin lifetime is long enough to become comparable to the interband recombination time, which is typically dominated by Auger processes for NGSs at $300 \mathrm{~K}$. We have greatly improved both the sensitivity and reliability of the method by introducing a photo-elastic modulator (PEM). This device sinusiodally varies the refractive index of a transparent solid, and hence, in conjunction with linearly polarised light at $45^{\circ}$, directly modulates between right and left circularly polarised light.

We previously demonstrated that spin flip (EY) scattering dominates in $\mathrm{Hg}_{0.78} \mathrm{Cd}_{0.22} \mathrm{Te}$ due to strong lattice scattering at $300 \mathrm{~K}$ [6]. In contrast, spin unphasing (DP) controls the loss of polarisation in intrinsic InSb and InAs due to the higher mobility, giving spin lifetimes, $\tau_{\mathrm{s}}$, of $\sim 20 \mathrm{ps}$ at $300 \mathrm{~K}[6,7]$. We found that heavy $\mathrm{n}$-doping of InSb and InAs introduced a long tail to the pump-probe data giving lifetimes more characteristic of the EY mechanism. We show here, by more careful measurements with the PEM, that the fast decay component is still present (although somewhat faster) at the same time as the long decay - i.e. there is a 2-component decay. Our samples, when at $300 \mathrm{~K}$, both have their chemical potential near to the band edge and are in the intermediate regime between nondegeneracy and strong degeneracy (see equations (1) and (2), next section). We would therefore expect a change in the relaxation rate due to DP somewhere betwen the predictions of equations (1) and (2). Using the measured carrier concentrations and mobilities, we find a predicted increase in the rate between 1.2 and 7 , which is consistent with the experiment. The long decay component has a lifetime that is consistent with EY, and it appears that both can contribute in these materials. 
We also report the first measurements of spin lifetimes in InSb/lnAISb quantum wells, grown at QinetiQ, of width $20 \mathrm{~nm}$ and doping concentration $2.5 \times 10^{11} \mathrm{~cm}^{-2}$. We see a very fast component at times less than $1 \mathrm{ps}$, which we attribute to thermalisation of the optically injected carriers, to hole spin relaxation, and to coherent artifacts associated with gratings written by the pump and probe beams (c.f. [8]). At times longer than $1 \mathrm{ps}$ spin polarisation decays via the DP process, with a lifetime of $\sim 17 \mathrm{ps}$, and this is followed by a long component of $400 \mathrm{ps}$. We ascribe this long component to the EY process which is less sensitive than the DP process to fast emptying of carriers by Auger recombination.

We have investigated the effect of injection of hot spinpolarised electrons high in the band (which may be important for electrical injection through a tunnel barrier, required in an actual spintronic device) and consequent polarisation decay as they lose energy. This was done by pumping with near infrared circularly polarised light pulses and probing with polarised light near the band-edge wavelength. The results show that for InSb, when pumping at $2 \mu \mathrm{m}$, about $10 \%$ of the spin polarisation is left by the time the electrons reach the bottom of the band.

Finally, we have performed far-infrared circular pump, mid-IR probe experiments to examine the role of holes (produced by interband photo-pumping in the degenerate pump/probe experiment) in the n-type doped sample. In this case the excitation, at $11 \mu \mathrm{m}$, is by circular Drude absorption, and spin polarises the existing electron population [14]. The induced Faraday rotation of the interband probe measures the spin population. It appears that very similar lifetimes are observed as for the degenerate interband experiment, and that the photoholes do not play a dominant role.

\section{Theory and discussion}

In bulk semiconductors with the zinc-blende structure the lack of inversion symmetry (bulk inversion asymmetry, BIA) allows a splitting via the spin-orbit interaction of the spin up and spin down energy levels, called the Dresselhaus spin splitting. In the conduction band of III-V semiconductors the splitting is proportional to $k^{3}$ for small linear momentum $k$, and gives rise to the DP spin unphasing mechanism.

The DP relaxation rate for lattice scattering and non-degenerate statistics is:

$$
\frac{1}{\tau_{s}}=\text { const. } \beta^{2} \frac{(k T)^{3}}{\hbar^{2} E_{\mathrm{g}}} \tau_{\mathrm{p}},
$$

where $\beta \approx\left(4 \gamma m^{*} / \mathrm{m}_{0}\right) /(3-\gamma)^{1 / 2}$ and $\gamma=\Delta /\left(\mathrm{E}_{\mathrm{G}}+\Delta\right)$. For the case of degenerate statistics and ionised impurity scattering the DP rate becomes:

$$
\frac{1}{\tau_{S}}=\text { const. } \beta^{2} \frac{\left(E_{F}\right)^{3}}{\hbar^{2} E_{G}} \tau_{p}
$$

As discussed above, equations (1) and (2) give good agreement with the results obtained experimentally for bulk $n-I n S b$ and $n-$ InAs.

Quantum structures give rise to the possibility of introducing further, structural inversion asymmetry (SIA) and a second, socalled Rashba, spin splitting mechanism becomes important $[9,10]$. The origin of the Rashba spin-orbit splitting is asymmetry in the confining potential that is present at the 2dimensional electron gas. This may be controlled by a gate voltage in spintronic applications. For [001] grown wells the Hamiltonian is $\hat{H}=\hbar^{2} k^{2} / 2 m^{*}+\hat{H}_{S O}$ and the spin-orbit part is:

$$
\hat{H}_{S O}=\alpha\left(\sigma_{x} k_{y}-\sigma_{y} k_{x}\right)+\beta\left(\sigma_{x} k_{x}-\sigma_{y} k_{y}\right) .
$$

where $\sigma$ are the Pauli spin vectors, $\alpha$ is the Rashba parameter and $\beta$ is the Dresselhaus parameter. The bulk Dresselhaus $k^{3}$ terms have been replaced by terms like $\left\langle k_{z}\right\rangle^{2} k_{x}$ and are now linear in $k$. The conduction band dispersion is anisotropic, and the average spin splitting at the Fermi energy for a degenerate electron gas is approximately (assuming $\alpha>\beta>0$ )

$$
\Delta \approx 2 \alpha k_{F} \text {, }
$$

where $k_{F}$ is the Fermi wavenumber. The Rashba effect is enhanced for NGSs due to the larger spin-orbit coupling term. Spin dynamics in quantum wells clearly differs from that in the bulk. The rate of precession is given by a combination of the Dresselhaus and Rashba terms. In particular the Rashba effect allows control of the spin precession via its contribution to $\mathbf{B}_{\text {eff }}$. The direction of $\mathbf{B}_{e f f}$ also depends on the crystal symmetry, and it has been shown that in quantum wells where the growth direction is $z / /[110], B(B I A)$ is along $z$, so $z$-polarised spins do not precess and hence do not unphase unless SLA is introduced. For example in GaAs/AlGaAs, [110] growth allows gatecontrol of spins in the particular case where they are polarised parallel to the growth direction, and recently this has been demonstrated in GaAs/AlGaAs wells at $170 \mathrm{~K}$ [11]. On the other hand, for [001] growth it has been noted that if the Rashba effect can be tuned with a gate voltage to be exactly equal in strength to the Dresselhaus term $(\alpha=\beta)$, then the eigenstates of $H$ are also eigenstates of spin, polarised in-plane in the [1-10] direction for all $\mathbf{k}$ [12-14]. Spins injected in this polarisation would not precess (and EY would also be forbidden due to lack of mixing) until $\alpha$ is tuned away from $\beta$. That work showed that even in GaAs/AlGaAs where $\beta>\alpha$ (and exact resonance is not achievable with electric field) a suppression of an order of magnitude in $\tau_{s}$ is possible. In NGS In $\mathrm{As} / \mathrm{AlGaSb}$ where $\beta<\alpha$ a suppression of nearly three orders was predicted, with wide tunability over a small range of fields. This could be the basis of a non-ballistic spin FET[13]. This encapsulates the primary reason for interest in [001] grown NGS wells for spin control: although the spin-splitting in the "off" state is large and hence the DP lifetime is short, the large value of $\alpha$ enables very strong quenching of this process in the "on" state.

This work is supported by the UK-EPSRC grant GR/R42402 and the EU contract FENIKS: G5RD-CT-2001 00535.

\section{References}

[1] S Datta and B Das, Appl. Phys. Lett. 56, 665 (1990).

[2] H Ohno, Science 281, 951 (1998).

[3] J M Kikkawa and D D Awschalom, Phys. Rev, Lett., 80, 4313 (1998).

[4] M I D'yakonov and V I Perel, Sov. Phys. JETP 33, 1053 (1971); Sov. Phys. Solid State 13, 3023 (1972).

[5] R J Elliott, Phys. Rev. 96, 266 (1954).

[6] P Murzyn et.al., Phys. Rev. B67, 235202 (2003)

[7] P Murzyn et. al., Appl. Phys. Lett., 83, 5220 (2003).

[8] T F Boggess et. al., Appl. Phys. Lett., 77, 1333 (2000).

[9] Y A Bychkov and E I Rashba, J. Phys. C17, 6039 (1984).

[10] W Zawadzki and P Pfeffer, Semicond. Sci. and Tech. 19, R1 (2004).

[11] O Z Karimov et. L., Phys. Rev. Lett., 91, 246601 (2003).

[12] J Kainz et. al., Phys. Rev. B68, 075322 (2003).

[13] J Schliemann et. al., Phys. Rev. Lett. 90, 146801 (2003).

[14] S D Ganichev, I N Yassievich and W Pretl, J. Phys. Condens. Matter 14, R1263 (2002). 